\title{
CONSTRUCTION COST PREDICTION USING NEURAL NETWORKS
}

\author{
Smita K. Magdum ${ }^{1}$ and Amol C. Adamuthe ${ }^{2}$ \\ ${ }^{I}$ Department of Computer Science and Engineering, Rajarambapu Institute of Technology, India \\ ${ }^{2}$ Department of Information Technology, Rajarambapu Institute of Technology, India
}

\begin{abstract}
Construction cost prediction is important for construction firms to compete and grow in the industry. Accurate construction cost prediction in the early stage of project is important for project feasibility studies and successful completion. There are many factors that affect the cost prediction. This paper presents construction cost prediction as multiple regression model with cost of six materials as independent variables. The objective of this paper is to develop neural networks and multilayer perceptron based model for construction cost prediction. Different models of $N N$ and MLP are developed with varying hidden layer size and hidden nodes. Four artificial neural network models and twelve multilayer perceptron models are compared. MLP and NN give better results than statistical regression method. As compared to NN, MLP works better on training dataset but fails on testing dataset. Five activation functions are tested to identify suitable function for the problem. 'elu' transfer function gives better results than other transfer function.
\end{abstract}

Keywords:

Construction Cost Prediction, Neural Network, Multilayer Perceptron

\section{INTRODUCTION}

Success of construction projects is examined by meeting to budget, timing, and quality of work as per owner's expectations. Construction manager or contractor needs effective tools for budget or cost estimation and work scheduling. Budget or cost prediction in early-stage plays a very important role in any construction project. An incorrect budget or cost forecasting can easily turn an estimated profit into loss [1]. Cost estimation of construction projects is a difficult problem because it is affected by many variable factors [2]. There are number of categories that can have major impacts on project costs. Such factor include the cost of materials, transportation charges, site condition, the size of the project, schedule of the project etc. [3] [4]. From those factors one of the most important factor is materials cost which affect the total construction cost.

The construction cost prediction problem is formulated as multivariable problem and experimented with methods such as regression [5], artificial neural network [3] and support vector machine [6]. These estimation methods, use some historical data of cost and find a functional relationship between change in cost and the factors on which the cost is depended. The main issue of cost estimates in construction projects includes the detailed project information, changes in design parameters, uncertainties regarding project development etc. Linear regression analysis shows little success. The statistical methods and regression analysis are used conventionally in literatures for cost estimation. All traditional methods [1] have limitations in accurate project cost prediction due to the large number of significant variables and interactions between these variables. Artificial intelligence approaches such as neural networks, evolutionary algorithms, fuzzy logic and hybrid methods are applicable to cost estimation or prediction problems [1]. During 1990s neural network [5] appeared as a viable alternative for estimating construction cost. The NNs [5] are a good alternative for construction costs prediction because this method eliminates the need to find a good cost estimating relationship that mathematically describes the cost of a system as a function of the variables that have the most effect on the cost of that system. In [4] [5], [7] [8] artificial neutrals network (ANN) approach is used for prediction of construction cost.

ANN has the ability to deal with the complex and nonlinear interaction between input and the outcome to be predicted. Earlier research has shown that the neural network model for cost estimation is better than traditional regression methods. ANN model proved that neural networks are able to reduce uncertainties related to the cost of construction projects. Finally, the further research seeks to develop a proper and realistic model for accurately estimating construction costs [4]. Accuracy of NN models depends on architecture of the model. Two main parameters in $\mathrm{NN}$ architecture are

- No of nodes in hidden layer: Less number of hidden neuron cause poor training while too may many hidden neurons in hidden layer leads over fitting problem.

- Transfer Function: Each hidden node and output node applies transfer function to input patterns. The selection of transfer functions may strongly impact performance of neural networks.

The purpose of this study is to design construction cost prediction model considering materials cost as input parameters. This paper shows cost prediction experimentation with two supervised learning algorithms, namely the neural network (NN) and Multilayer Perceptron (MLP).The objective of the paper is to identify best NN and MLP model with suitable activation functions, hidden layers and hidden neurons.

The next section briefly describes background of cost estimation problem and different problems solved using different prediction methods. Section 3 describes construction cost prediction models with NN and MLP techniques. Section 4 describes the experimental result by considering different parameters. To end, in section 5 presents conclusions of our work.

\section{RELATED WORK}

Prediction of construction cost estimation involves so lots of multivariate statistical methods. Linear regression models and artificial neural network models are used to predict the cost in construction projects such as apartments [4], buildings [1], [3], [6]-[7] and roads [5], [9]. 
Kim et al. [5] experimented three algorithms namely regression, neural network and case based reasoning for cost estimation of road construction projects. The models are based on year, gross floor area, storeys, total unit, duration, roofs types, FND types, usage of basement, finishing grades as input parameters and actual cost as output parameter. Proposed 75 neural network models are measured using estimation error. Proposed 75 neural network models are presented with varying parameters such as number of neurons in hidden layer, learning rate and momentum. Best results are obtained by NN model with 12-25-1(0.6-0.6), where $12,25,1,0.6$ and 0.6 are the input neurons, hidden neurons, output neurons, learning rate and momentum respectively.

Kim et al. [6] presented three algorithms namely Regression, Neural Network and Support Vector Machine for cost estimation of building construction projects. The model is based on year, budget, school levels, land acquisition, class number, building area, gross floor area, storey, basement floor and floor height as input parameters and total construction cost as output parameter. Proposed neural network model is measured using actual error rate, mean absolute error (MAER) and standard deviation. MAER of three results are then compared using analysis of variance (ANOVA). Best results are obtained by $\mathrm{NN}$ model with 5.27 MAER and 4.13 standard deviation.

Mahamid and Bruland [9] developed multiple and linear regression for cost prediction of road construction projects. The model is based on road length $(\mathrm{km})$, pavement width $(\mathrm{km})$, pavement thickness after compaction, haul distances, pavement area as input parameters and total cost of asphalt works, the second is the cost $/ \mathrm{m}$, and the last one is the cost $/ \mathrm{m}^{2}$ as output parameter. Proposed multiple linear regression model are measured using coefficients of determination $\left(r^{2}\right)$, p-value and $\mathrm{F}$ value. Best results of multiple and linear regression model with coefficient of determination ranging from 0.57 to 0.96 and $p$-value for each model is less than 0.05 which means that the use of dependent variable in the model is significant.

In [1], authors presented evolutional fuzzy hybrid neural network cost estimation for building construction projects. The proposed model uses impact factors from seven engineering categories. Proposed evolutional fuzzy hybrid neural network (EFHNN) model incorporates four artificial intelligence approaches such as neural network, high order neural network, fuzzy logic, and genetic algorithm. Performance is measured using estimation error. Proposed EFHNN models is presented with varying parameters such as number of neurons, number of hidden layer, activation function, crossover rate and mutation rate.

Cheng et al. [3] experimented integrated rough set theory and artificial neural network for cost prediction of building construction projects. The model is based on total height, standard layer area, type of structure, project management level, period, basement area as input parameters and building construction cost as output parameter. Proposed neural network models are measured using estimation error. Proposed neural network model are presented with varying parameters such as number of neurons in hidden layer, learning rate and expectative error. Best results are obtained by $\mathrm{NN}$ model with $6-5-1$, where 6,5 and 1 are the input neurons, hidden neurons and output neurons, respectively with performance of 0.0009956 , where expectative error is 0.001 with training time only 0.405 seconds.
Luu and Kim [4] experimented neural network for cost prediction of apartment construction projects. The model is based on storey, total area, building level, year, gasoline cost, steel cost, cement cost as input parameters and total cost of building as output parameter. Proposed neural network model is measured using mean percentage error (MPE) and mean absolute percentage error (MAPE). Proposed neural network models are with varying parameters such as number of neurons, number of hidden layer, activation function and adaption learning function. Result shows that neural network has potential to improve the cost estimation model for apartment projects.

Günaydın and Doğan [7] proposed neural network model for cost estimation of structural systems of buildings construction projects. The model is based on total area of the building, ratio of the typical floor area to the total area of the building, ratio of ground floor area to the area of building, number of floor, console direction of the building, foundation system of the building, floor type of building, location as input parameters and cost of the structural system per square meter as output parameter. Proposed neural network models are measured using cost percentage error (CPE) and mean square error (MSE). Proposed neural network models are presented with varying parameters such as number of neurons in hidden layer, learning rate and momentum. Best results are obtained by NN model with 8-4-1, where 8,4 and 1 are the input neurons, hidden neurons and output neurons respectively.

Deep-learning methods are machine learning algorithms [10] with multiple levels of representation are representation-learning methods, which are obtained by composing simple modules that each transforms the representation at one level into a representation at a higher, slightly more abstract level [11]. There are supervised learning algorithms namely recurrent network [12], convolutional neural network [13] and multilayer perceptron [14]. In [14] [17], authors presented multilayer perceptron (MLP) for prediction problems such as breast cancer [14], wind forecasting [14] and heart disease [16] [17].

\section{CONSTRUCTION COST PREDICTION MODEL}

This section describes neural network based approach for construction cost prediction. Methodology developed to achieve the objectives of this paper is presented in Fig.1. There two main phases.

i) Identify independent variables and collect historic dataset

ii) Designing phase (NN and MLP)

The designing phase includes the design of the neural network architecture. It is a complex and dynamic process that requires the determination of the type of activation function, number of hidden layers and neurons.

\subsection{MULTIPLE REGRESSION MODEL}

Accurate estimation of quantities and costs is a crucial factor in success of construction projects. The main complexity in the cost prediction is dependency on several factors. From those factors one of the most important factors is materials cost. The material cost is main impact factor in construction project for cost estimation which is varying every year. Cost of cement, sand, steel, aggregates, mason, skilled worker, non-skilled work worker 
is the major factors which are considered in cost estimation. As shown in Table. 1 six input and one output variables are extracted from the collected dataset [2] in which data of last twenty-three years has been collected from Schedule of rate book (SOR) and general studies.

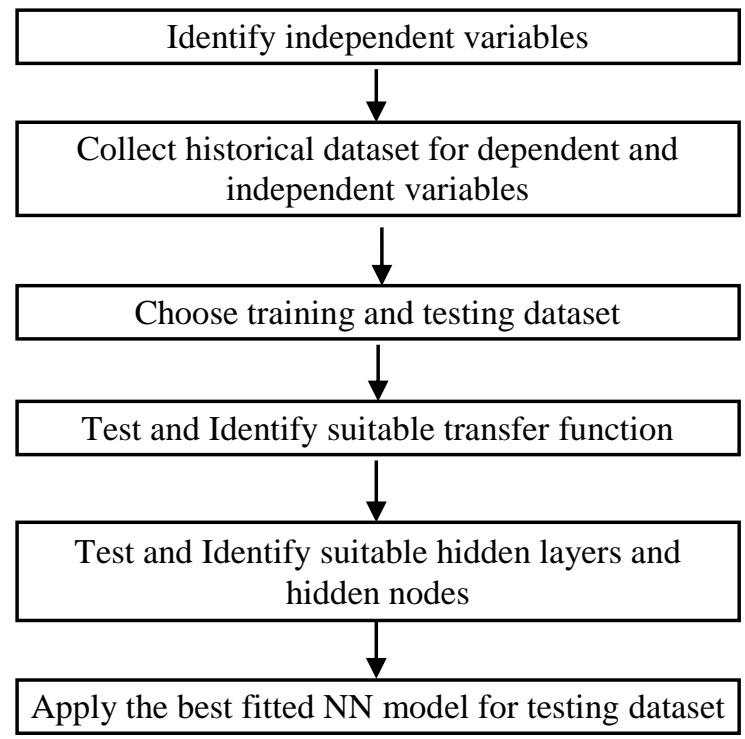

Fig.1. Methodology for Construction Cost Prediction

Table.1. Input and output variables

\begin{tabular}{|c|c|c|}
\hline Description & \multicolumn{2}{|c|}{ Unit } \\
\hline \multirow{4}{*}{ Input Variable } & Cement & $(\mathrm{Rs} / \mathrm{bag})$ \\
& Sand & $(\mathrm{Rs} / \mathrm{ft} 3)$ \\
& Steel & $(\mathrm{Rs} / \mathrm{kg})$ \\
& Aggregate & $(\mathrm{Rs} / \mathrm{ft} 3)$ \\
& Mason & $(\mathrm{Rs} /$ day) \\
& Skilled Labor & $(\mathrm{Rs} /$ day $)$ \\
\hline Output Parameters & Contractor Cost & $(\mathrm{Rs} / \mathrm{ft} 2)$ \\
\hline
\end{tabular}

Regression analysis is a tool which defines the interaction between variables. The analyzer process the statistical significance of the predictable relationships, that is, the degree of assurance that is the relationship between predictable relationships [20]. Regression is used for time series prediction, forecasting technique and finds the relationship between the dependent and independent variables. Regression is used for modeling and analysis of data. Multiple regressions defined as the relationships between one output variable and two or more than two predictors. In [5], multiple regression is represented as in Eq.(1).

$$
Y=A_{0}+A_{1} \cdot X_{1}+A_{2} \cdot X_{2} \ldots+A_{n} \cdot X_{n}+\text { error }
$$

where, $Y$ is construction cost and $X_{1}, X_{2}, \ldots, X_{n}$ are material costs.

\subsection{NEURAL NETWORK}

The problem presented in this paper is based on feed-forward neural network architecture and back-propagation learning technique.

An artificial neural network generally called neural network is a computational model which is used to modelling non-linear statistical data in order to model complex relationships between inputs and outputs. Their high performance in modelling relationships between inputs and outputs make neural networks reliable tools, which can also be used in the development of cost estimation models [18]. The structure of NN, which consists of three basic layers, input, hidden and the output layer. Each one contains several neurons except output layer; it contains one neuron that represents the output of training process. Cost estimation model in the construction project, which depends on an artificial neural network that adapts to cost estimation better in construction project [8]. The NN [7] can be formulated as in Eq.(2)

$$
y=f(n e t)=f\left(\sum_{i=1}^{n} w_{i} x_{i}+b\right)
$$

where, $n$ is the number of inputs, $x_{i}$ is the inputs given to the processing element, $w_{i}$ are the weights and $b$ is a bias term. $f$ is activation function. The activation function $f$ performs a mathematical operation on the signal output.

\subsection{MULTILAYER PERCEPTRON}

Multilayer perceptron (MLP) network is feed-forward neural network containing multiple hidden layers. The single layer perceptron is able to solve linearly separable problems. When multiple layers are added into single layer perceptron to solve the complex problem which is not linearly separable this model is called as multilayer perceptron [14].

An MLP neural network consists of a number of interconnected artificial neurons which is the basic processing element of a neural network that is connected only in a forward manner to form layers. It consists of a linear combiner followed by a transfer function. MLP formulation is taken from the paper [16] and [19].

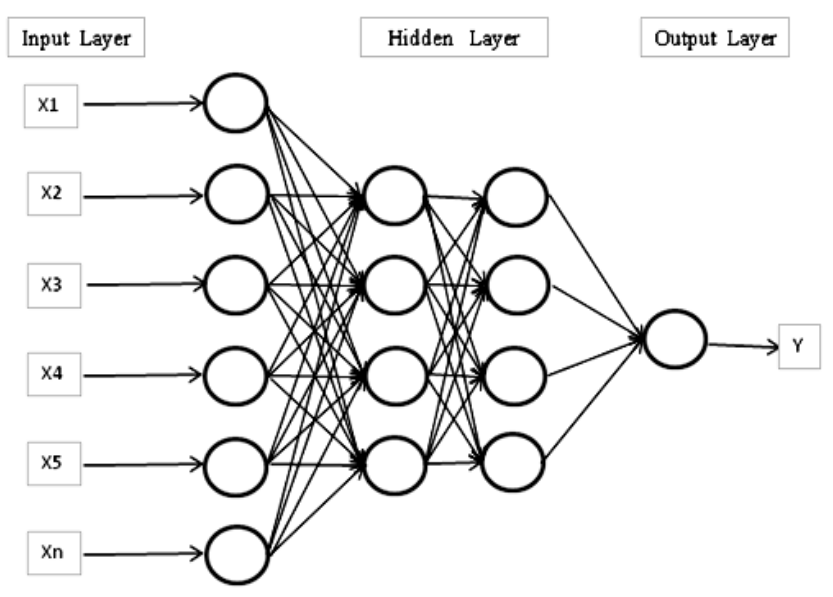

Fig.2. Multi-layer Perceptron Architecture [18]

$$
\begin{aligned}
& y_{k}^{(n)}=f^{(n)}\left(\sum_{j} y_{j}^{(n-1)} \cdot w_{j k}^{(n)}\right) \\
& y_{k}^{(n-1)}=\sum_{i} x_{i}^{(n-1)} \cdot w_{i j}^{(n)}+b_{i j}^{(n)}
\end{aligned}
$$

Each unit $j$ in layer $n$ receives activations $y_{j}^{(n-1)} \cdot w_{j k}^{(n)}$ from the previous layer of processing units and sends activations $y_{k}^{(n)}$. Here, $y_{k}^{(n)}$ is predicted rate, $f^{(n)}$ is activation function $x_{i}^{(n-1)}$ is rate of materials, $w_{i j}^{(n)}$ and $w_{j k}^{(n)}$ is connection weights between the 
material rate and the hidden neuron and between the hidden neuron and the predicted cost, $b_{i j}^{(n)}$ is bias terms and $i, j$ and $k$ are the number of neurons in each layer. An exponential linear unit transfer function is used in this study for its special properties. An exponential linear unit transfer function is taken from [19].

$$
f(x)=\left\{\begin{array}{cc}
x & x>0 \\
\alpha(\exp (x)-1) & x \leq 0
\end{array}=\left\{\begin{array}{cr}
1 & x>0 \\
f(x)+\alpha & x \leq 0
\end{array}\right.\right.
$$

where, $x$ is the input to a neuron. ELU activations are the simplest non-linear activation function. ELU speeds up learning model in deep neural networks and gives higher accuracies than other activation function.

\section{EXPERIMENTAL DISCUSSION \\ RESULTS}

AND

Number of hidden layers, number of hidden nodes, activation function and learning rate has impact on the performance of neural network. Different artificial neural network and multilayer perceptron models are developed by varying parameters: number of hidden layers, number of hidden nodes and activation function. This section presents the proposed models, results and discussion.

The proposed model for prediction of cost estimation problem using neural network is implemented in Python version 3.5 using the TensorFlow framework with Keras library. Keras is the library used to develop deep learning model with TensorFlow as backend.

The best way to evaluate the forecast model is compare its actual and predicted results. To compare the performance of models Root Mean Square Error (RMSE) is used as a measure.

$$
R M S E=\sqrt{\frac{1}{n} \sum_{i=1}^{n}(\text { Predicted cost }- \text { Actual cost })^{2}}
$$

The performances of activation functions are experimented by keeping the two hidden layers in MLP model with 10 and 8 neurons in each layer and single layer in NN model with 10 neurons. The Table. 2 shows the RMSE values. In both models, $e l u$ activation function gives minimum error as compared to other activation functions. MLP with elu activation function gives minimum error than NN model.

Table.2. RMSE values of Training Model for Activation Functions

\begin{tabular}{|c|c|c|c|c|c|c|}
\hline Algorithms & Epoch & $\mathbf{5 0 0}$ & $\mathbf{1 0 0 0}$ & $\mathbf{1 5 0 0}$ & $\mathbf{2 0 0 0}$ & $\mathbf{2 5 0 0}$ \\
\hline \multirow{4}{*}{ NN } & Relu & 0.68 & 0.41 & 0.32 & 0.28 & 0.21 \\
\cline { 2 - 7 } & Softmax & 7.65 & 5.69 & 4.16 & 2.95 & 1.98 \\
\cline { 2 - 7 } & Tanh & 1.24 & 0.32 & 0.11 & 0.01 & 0.05 \\
\cline { 2 - 7 } & Sigmoid & 3.37 & 1.29 & 0.44 & 0.24 & 0.13 \\
\cline { 2 - 7 } & Elu & 0.38 & 0.24 & 0.2 & 0.12 & 0.03 \\
\hline \multirow{4}{*}{ MLP } & Relu & 0.4 & 0.2 & 0.1 & 0.04 & 0.02 \\
\cline { 2 - 7 } & Softmax & 7.29 & 5.43 & 4.04 & 2.78 & 1.87 \\
\cline { 2 - 7 } & Tanh & 1.73 & 0.43 & 0.08 & 0.07 & 0.04 \\
\cline { 2 - 7 } & Sigmoid & 4.41 & 1.43 & 0.44 & 0.14 & 0.03 \\
\cline { 2 - 7 } & Elu & 0.23 & 0.12 & 0.04 & 0.01 & 0.01 \\
\hline
\end{tabular}

The NN and MLP experimentation is carried out by dividing the dataset into two sets - training set and testing set. From total records $70 \%$ records are used for training and remaining $30 \%$ records are used for testing.

This model consists of an input layer with 6 nodes, and an output layer with one node. Paper [21] reported that, one hidden layer neural network is suitable for most application in construction. Our experiments are conducted with one hidden layer neural network. Number of neurons in the hidden layers is an important decision in developing neural network architecture. In paper [22] listed three rule-of-thumb for determining the correct number of neurons to use in the hidden layers.

i) The number of hidden neurons should be in the range between the sizes of the input layer and the size of the output layer.

ii) The number of hidden neurons should be $2 / 3$ of the input layer size, plus the size of the output layer.

iii) The number of hidden neurons should be less than double the input layer size.

These rules provide a starting point for experimentation of NN and MLP. The performances of NN are experimented by changing the hidden neuron 4, 6, 8, and 10. The Fig. 3 and Fig.4 shows the performance of NN models on training and testing dataset, respectively.

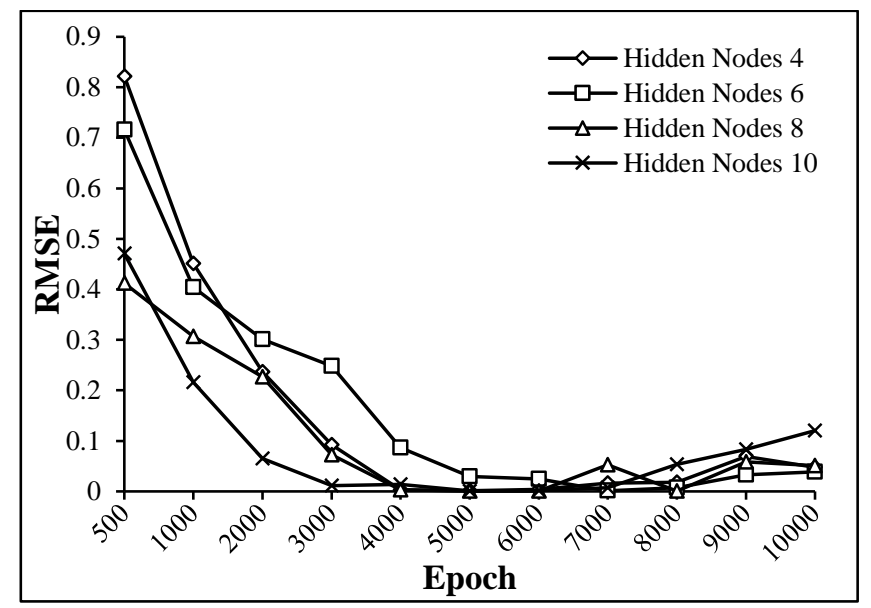

Fig.3. Performance of NN with hidden nodes 4, 6, 8 and 10 on training dataset

The Fig.3 and Fig.4 shows the RMSE values of single layer $\mathrm{NN}$ which are experimented by changing the hidden neurons 4,6 , 8 and 10 with respect to epochs. Error decreases with epochs up to a certain level. After that errors are slightly increased. The NN with eight hidden nodes gives best training and testing results. NN training model with eight hidden nodes at epoch 5000 gives the best result with RMSE value 0.0008 .

Multilayer perception model is designed with two hidden layers. We used two hidden layers in MLP model with different neurons in the combination of $\mathrm{XY}$ where, $\mathrm{X}$ denoted number of neuron in first layer and $\mathrm{Y}$ denoted number of neuron in second layer like 4-6, 4-8, 4-10, 6-4, 6-8, 6-10, 8-4, 8-6, 8-10, 10-4, 10-6 and 10-8. The Fig. 3 to Fig. 12 shows the performance of MLP models on training and testing dataset.

The Fig. 5 and Fig. 6 shows the RMSE values of two layer MLP which are experimented by changing the hidden neurons 4-6, 4-8 
and 4-10 with respect to epochs. Error decreases with epochs. MLP with 4-8 hidden nodes combination gives best result on training dataset. MLP with 4-10 hidden nodes combination gives better results on testing dataset. MLP training model with 4-8 hidden nodes at epoch 5000 gives the best result with RMSE value 0.0005 .

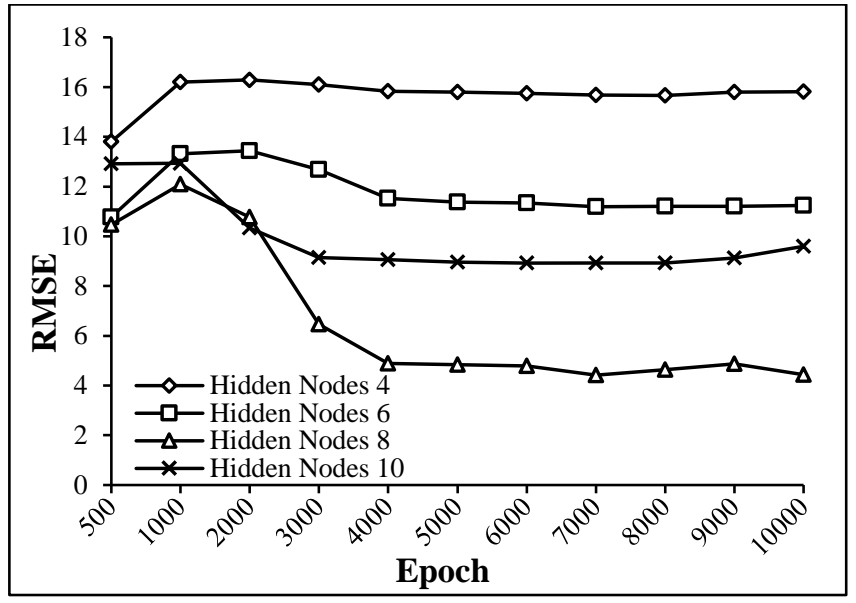

Fig.4. Performance of NN with hidden nodes 4, 6, 8 and 10 on testing dataset

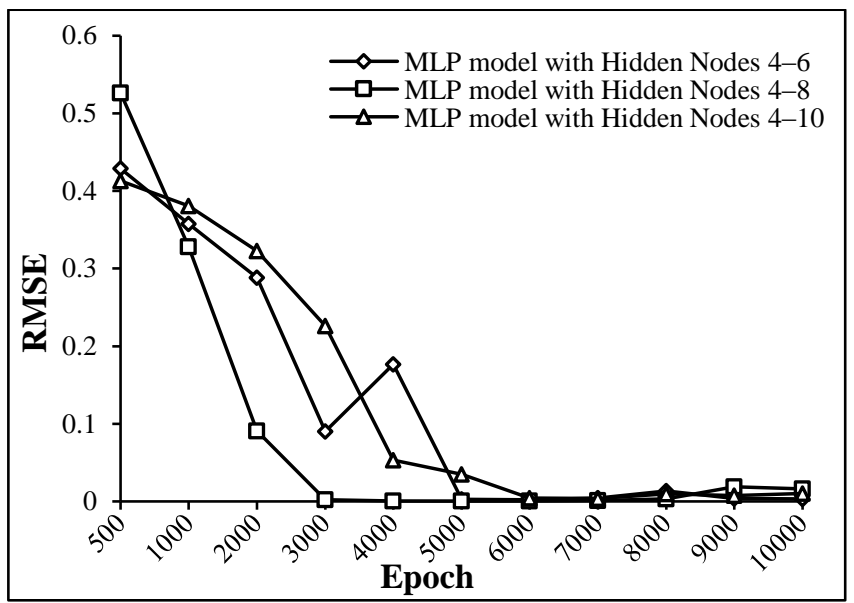

Fig.5. Performance of MLP models (4-6, 4-8 and 4-10) on training dataset

The Fig. 7 and Fig. 8 shows the RMSE values of two layer MLP which are experimented by changing the hidden neurons $6-4,6-8$ and 6-10 with respect to epochs. MLP with 6-4 hidden nodes combination shows fast convergence than other combinations.

The Fig.9 and Fig.10 shows the RMSE values of two layer MLP which are experimented by changing the hidden neurons 84, 8-6 and 8-10 with respect to epochs. MLP with 8-4 hidden nodes combination gives better results on testing dataset.

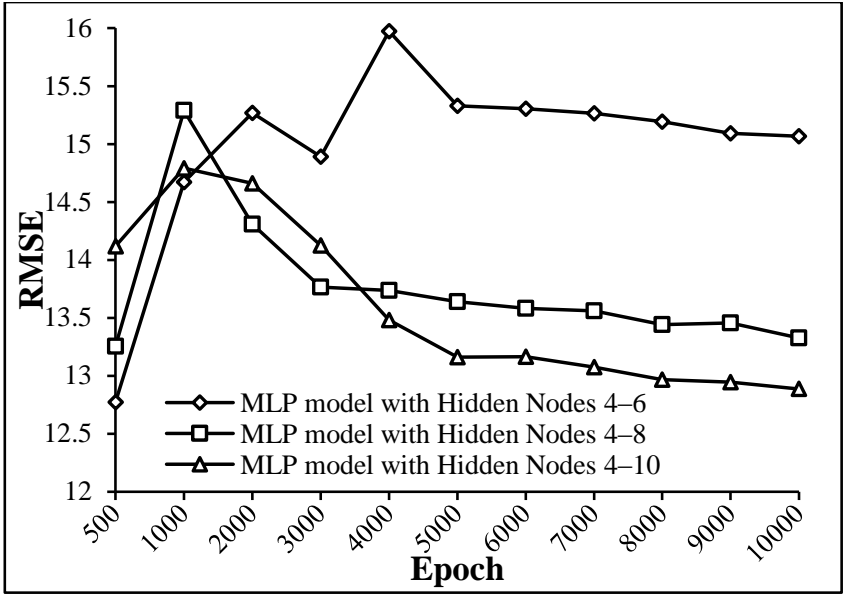

Fig.6. Performance of MLP models (4-6, 4-8 and 4-10) on testing dataset

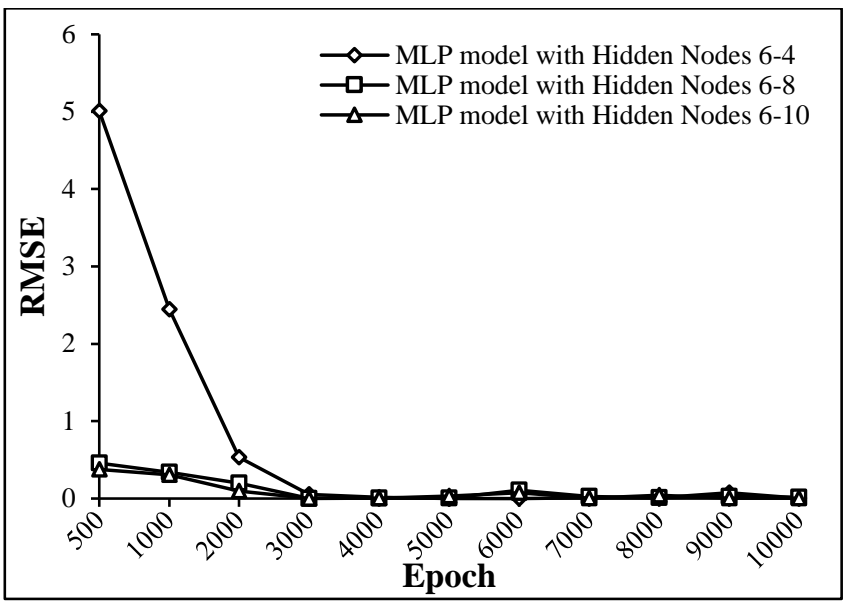

Fig.7. Performance of MLP models (6-4, 6-8 and 6-10) on training dataset

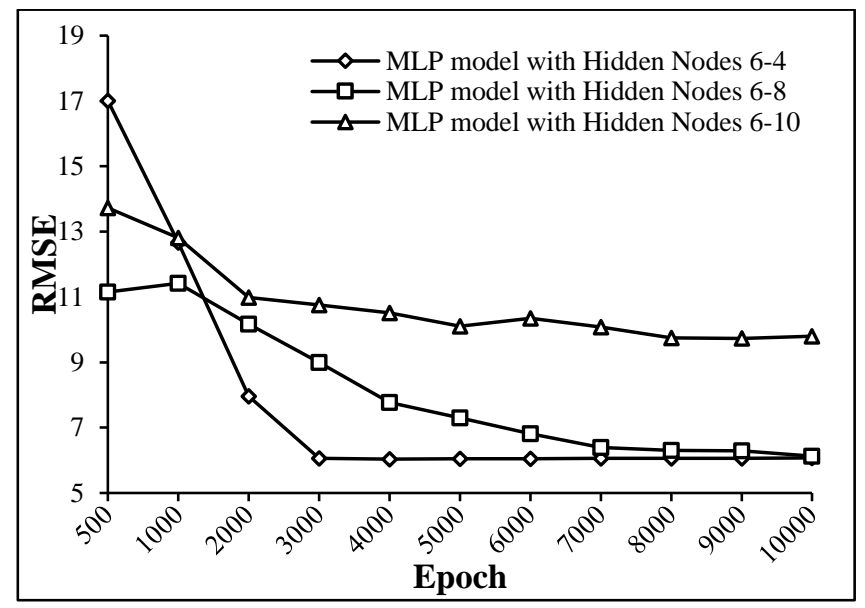

Fig.8. Performance of MLP models (6-4, 6-8 and 6-10) on testing dataset 


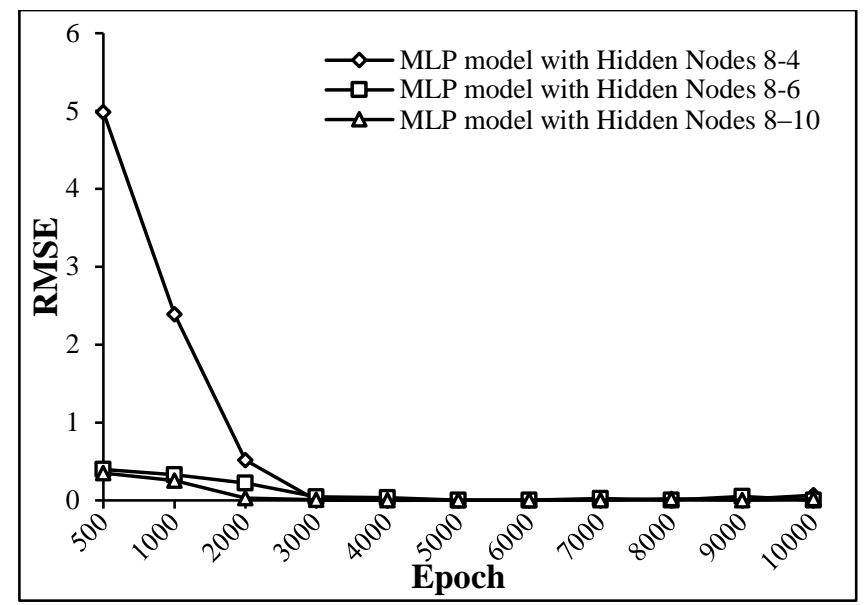

Fig.9. Performance of MLP models (8-4, 8-6 and 8-10) on training dataset

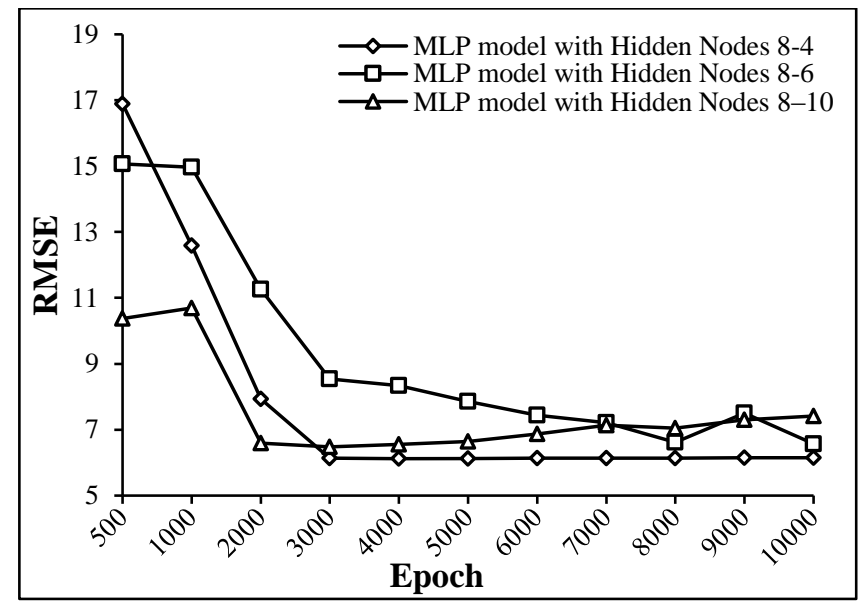

Fig.10. Performance of MLP models (8-4, 8-6 and 8-10) on testing dataset

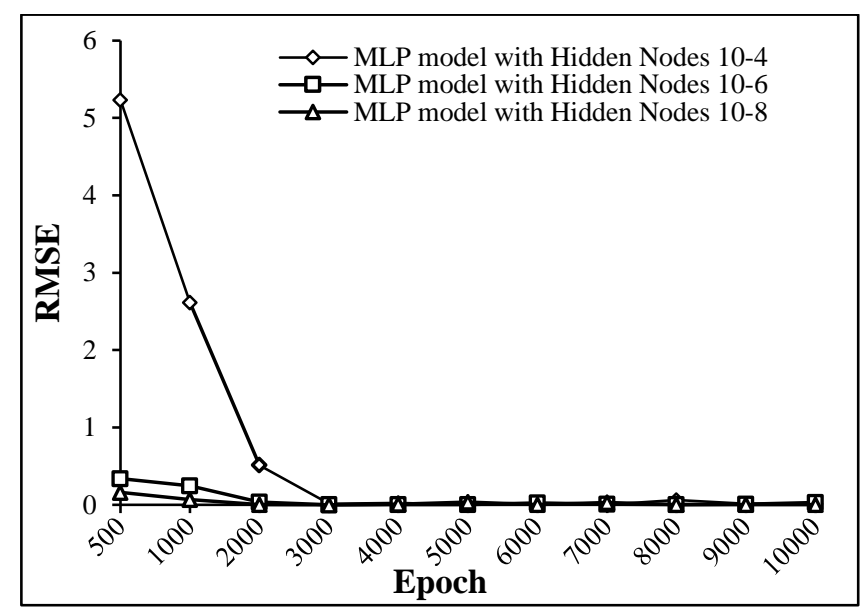

Fig.11. Performance of MLP models (10-4, 10-6 and 10-8) on training dataset

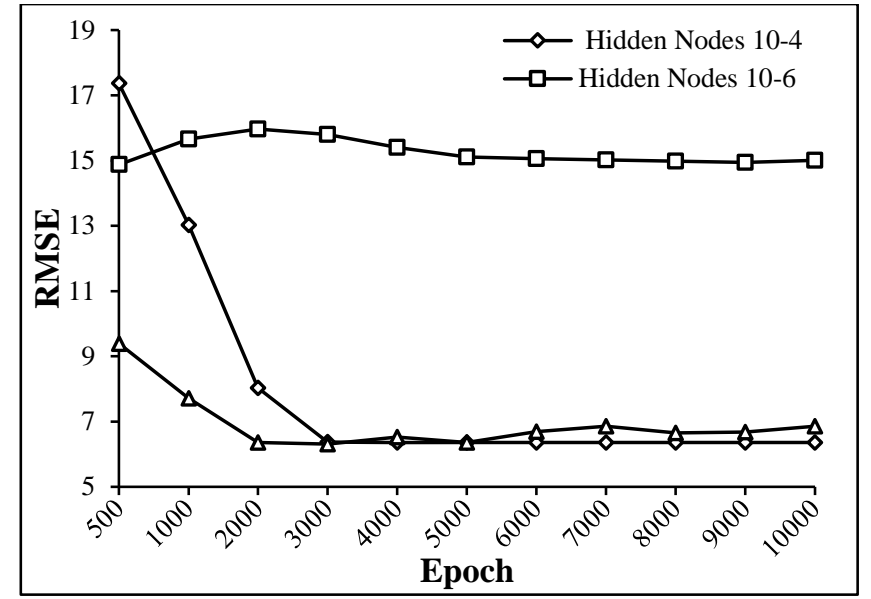

Fig.12. Performance of MLP models (10-4, 10-6 and 10-8) on testing dataset

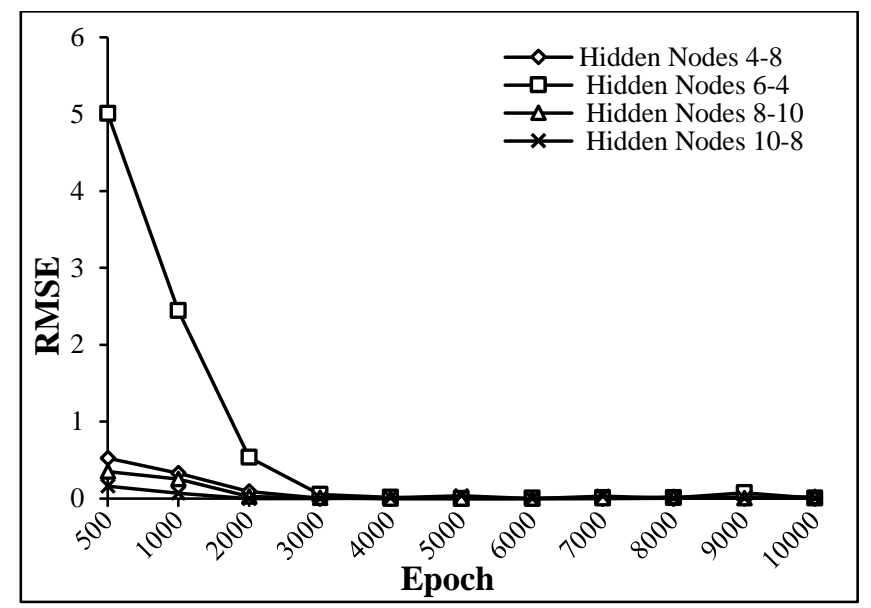

Fig.13. Performance of MLP models (4-8, 6-4, 8-10 and 10-8) on training dataset

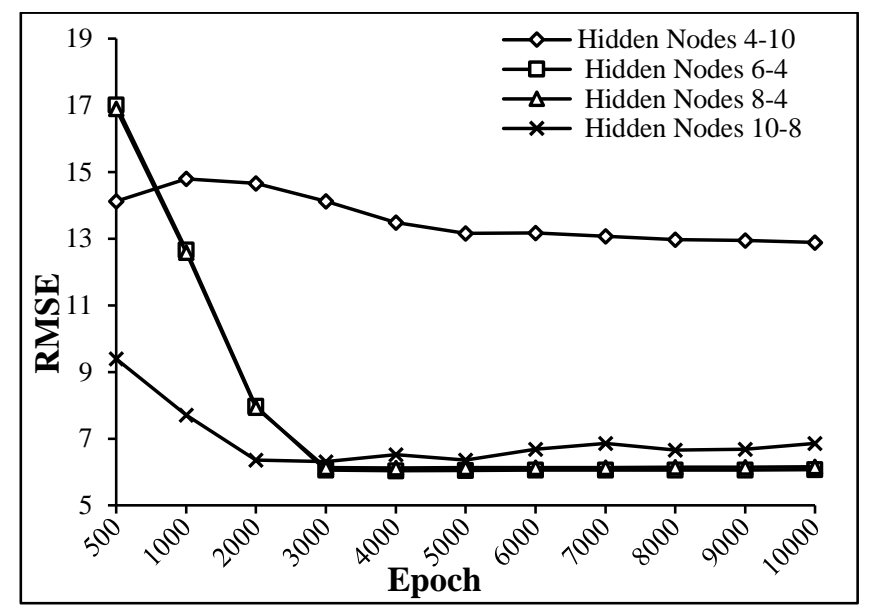

Fig.14. Performance of MLP models (4-10, 6-4, 8-4 and 10-8) on testing dataset 


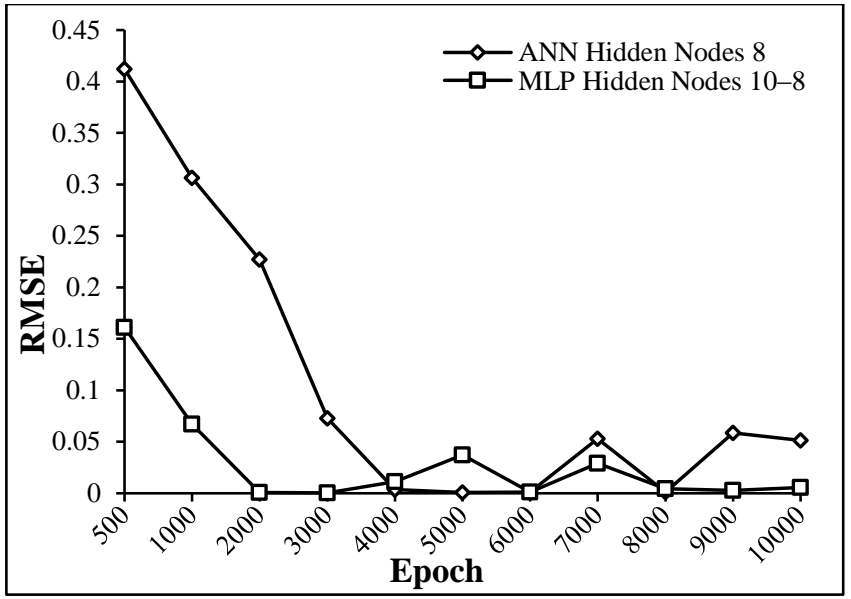

Fig.15. Comparison of best fitted NN and MLP models on training dataset

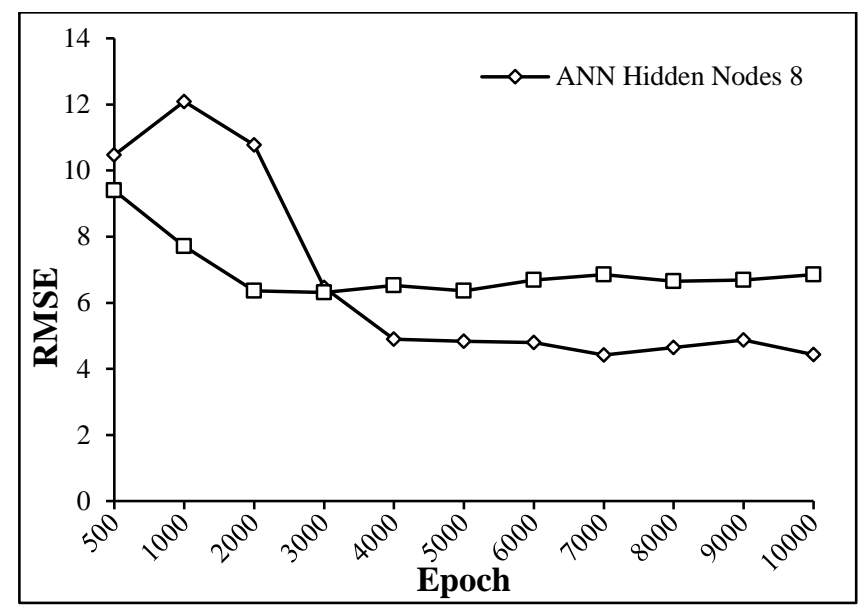

Fig.16. Comparison of best fitted NN and MLP models on testing dataset

The Fig.11 and Fig.12 shows the RMSE values of two layer MLP which are experimented by changing the hidden neurons 104, 10-6 and 10-8with respect to epochs. MLP with 10-4 and 10-8 hidden nodes combination are better than 10-6. MLP training model with 10-8 hidden nodes at epoch 3000 gives the best result with RMSE value 0.0004 .

The Fig.13 and Fig.14 shows the RMSE values of best MLP training results 4-8, 6-4, 8-10 and 10-8 from all the combination of hidden neurons with respect to epochs. Performance of NN model 4-10 is not suitable to the problem.

RMSE values of the proposed models are statistically different. Therefore, the best NN model and best MPL model performed are compared. The Fig.15 and Fig.16 shows comparison of best fitted NN and MLP models on training and testing datasets respectively. Performance of MLP model (10-8) is better than NN model with 8 hidden nodes on training dataset. But MLP models fails to give better results on testing dataset.

Performance of NN and MLP is compared with statistical multiple regressions model. Comparison is done on complete dataset. The RMSE values for multiple linear regressions, NN and MLP are 62.6269, 41.69 and 28.49 respectively. Performance of MLP is better than the NN and statistical multiple regression.

\section{CONCLUSION}

The outcome of our research is models for predicting the cost of construction projects. This study applied two techniques namely neural networks and multilayer perceptron for estimating construction costs. Four NN models and twelve MLP models are tested based on varying number of hidden layers and hidden nodes. Performance of NN on training and testing dataset with different hidden nodes varies significantly. Performance difference of MLP models on training dataset is not significant. MLP with ten and eight hidden nodes gives best training results. But, MLP models fail to give better results than NN with 8 hidden nodes on testing dataset.

These methods are compared with statistical multiple regression method. RMSE values of NN and MLP models are consistently low for training data set.

\section{REFERENCES}

[1] M.Y. Cheng, H.C. Tsai and E. Sudjono, "Conceptual Cost Estimates using Evolutionary Fuzzy Hybrid Neural Network for Projects in Construction Industry", International Journal on Expert Systems with Application, Vol. 37, No. 6, pp. 4224-4231, 2010.

[2] R. Yadav, M. Vyas, V. Vyas and S. Agrawal, "Cost Estimation Model for Residential Building using Artificial Neural Network", International Journal of Engineering Research and Technology, Vol. 5, No. 2, pp. 312-314, 2016.

[3] Huawang Shi and Wanqing Li, "The Integrated Methodology of Rough Set Theory and Artificial NeuralNetwork for Construction Project Cost Prediction", Proceedings of $2^{\text {nd }}$ International Symposium on Intelligent Information Technology Application, pp. 16-21, 2008.

[4] V.T. Luu and S.Y. Kim, "Neural Network Model for Construction Cost Prediction of Apartment Projects in Vietnam", Korean Journal of Construction Engineering and Management, Vol. 10, No. 3, pp. 139-147, 2009.

[5] G.H. Kim, S.H. An and K.I. Kang, "Comparison of Construction Cost Estimating Models based on Regression Analysis, Neural Networks, and Case-based Reasoning", Journal on Building and Environment, Vol. 39, No. 10, pp. 1235-1242, 2004.

[6] G.H. Kim, J.M. Shin, S. Kim and Y. Shin, "Comparison of School Building Construction Costs Estimation Methods Using Regression Analysis, Neural Network, and Support Vector Machine", Journal of Building Construction and Planning Research, Vol. 7, No. 1, pp. 1-7, 2013.

[7] H.M. Gunaydın and S.Z. Dogan, "A Neural Network Approach for Early Cost Estimation of Structural Systems of Buildings", International Journal of Project Management, Vol. 8, No. 22, pp. 595-602, 2004.

[8] M. Sana, I. Arazi, M. Faris Khamidi and Z. Saiful Bin, "Development of Construction Labor Productivity Estimation Model using Artificial Neural Network", Proceedings of IEEE National Postgraduate Conference, pp. 1-5, 2011.

[9] I. Mahamid and A. Bruland, "Preliminary Cost Estimating models for Road Construction Activities", Proceedings of 
International Conference on Critical Infrastructure Development, pp. 1-13, 2010.

[10] X. Qiu, L. Zhang, Y. Ren, P.N. Suganthan and G. Amaratunga, "Ensemble Deep Learning for Regression and Time Series Forecasting", Proceedings of IEEE Symposium on Computational Intelligence in Ensemble Learning, pp. 17, 2014.

[11] L. Deng and D. Yu, "Deep Learning: Methods and Applications", Foundations and Trends in Signal Processing, Vol. 7, No. 3-4, pp. 197-387, 2014.

[12] P.G. Madhavan, "Recurrent Neural Network for Time Series Prediction", Proceedings of IEEE $15^{\text {th }}$ annual International Conference of Engineering in Medicine and Biology Society, pp. 250-259, 1993.

[13] A. Krizhevsky, I. Sutskever and G.E. Hinton, "ImageNet Classification with Deep Convolutional Neural Networks", Proceedings of International Conference on Advances in Neural Information Processing Systems, pp. 1-9, 2012.

[14] M. Dalto, J. Matusko and M. Vasak "Deep Neural Networks for Time Series Prediction with Applications in Ultra-ShortTerm Wind Forecasting", Proceedings of IEEE International Conference on Industrial Technology, pp. 1$10,2015$.

[15] S.A. Mojarad, S.S. Dlay, W.L.Woo and G.V. Sherbet, "Breast Cancer Prediction and Cross Validation using Multilayer Perceptron Neural Networks", Proceedings of $7^{\text {th }}$ International Symposium on Communication Systems Networks and Digital Signal Processing, pp. 1-5, 2010.
[16] J.S. Sonawane and D.R. Patil, "Prediction of Heart Disease using Multilayer Perceptron Neural Network", Proceedings of IEEE International Conference on Information Communication and Embedded Systems, pp. 1-6, 2014.

[17] M. Durairaj and V. Revathi, "Prediction of Heart Disease using Back Propagation MLP Algorithm", International Journal of Scientific and Technology Research, Vol. 4, No. 8, pp. 235-239, 2015.

[18] D.A. Clevert, T. Unterthiner and S. Hochreiter, "Fast and Accurate Deep Network", Proceedings of International Conference on Learning Representations, pp. 1-14, 2016.

[19] John A. Bullinaria, "Learning in Multi-Layer PerceptronsBack-Propagation", Available http://www.cs.bham.ac.uk/ jxb/NN/17.pdf.

[20] Analytics Vidhya, "Types of Regression", Available at: https://www.analyticsvidhya.com/blog/2015/08/comprehen siveguide-regression/. Accessed on 2017.

[21] Hegazy T, P. Fazio and O. Moselhi, "Developing Practical Neural Network Applications using Back-Propagation", Computer Aided Civil and Infrastructure Engineering, Vol. 9, No. 2, pp. 145-159, 1994.

[22] P. Gaurang, G. Amit, Y.P. Kosta and P. Devyani, "Behaviour Analysis of Multilayer Perceptrons with Multiple Hidden Neurons and Hidden Layers", International Journal of Computer Theory and Engineering, Vol. 3, No. 2, pp. 332-337, 2011. 